\title{
Distribution of the Lactobacillus acidophilus Complex in Human Fecal Specimens Examined by Restriction Fragment Length Polymorphism of the 16S rRNA Genes
}

\author{
Yasuyuki Seto, Akira Kımura, Yoshihito Akal and Shigeru Fujıwara* \\ Technology and Research Institute, Snow Brand Milk Products Co., Ltd., Kawagoe, Saitama 350-1165, Japan \\ Received May 31, 2002; Accepted for publication, April 15, 2003
}

\begin{abstract}
Fresh fecal samples from healthy volunteers were examined for their content of the Lactobacillus acidophilus complex (LAC). A two-step isolation method for fecal lactobacilli was developed and employed in this study. Isolates of lactobacilli were identified according to their restriction fragment length polymorphism (RFLP) of genes coding for ribosomal RNA. Our results suggested that all samples contain lactobacilli and the most dominant species of human fecal lactobacilli is $L$. paracase $i$. In addition, several strains of $L A C$ were recovered from specimens of volunteers. Among six LAC species, namely, A1: L. acidophilus, A2: $L$. crispatus, A3: L. amylovorus, A4: L. gallinarum, B1: L. gasseri, and B2: $L$. johnsonii, isolates classified into cluster B were recovered from 6 out of 15 volunteers; however isolates of cluster $A$ were recovered from 3 of 15 volunteers. Four of 15 volunteers had strains of $L$. gasseri, 3 of them had strains of $L$. johnsonii, and strains of $L$. amylovorus were also isolated from specimens of 3 of 15 volunteers. These findings suggest that group B of LAC is the predominant cluster of LAC found in human feces and $L$. amylovorus was the only species of cluster $A$ found in this study. No volunteer with blood type $O$ had LAC in the fecal Lactobacillus flora.
\end{abstract}

Key words: PCR-RFLP; Isolation of fecal Lactobacillus; Distribution of LAC

\section{INTRODUCTION}

Bibel (2) reviewed the findings of Metchnikoff who had suggested in 1901 that the lactic acid bacteria inhabiting the gastrointestinal tract might prevent human diseases. Previously, Sandine (28) had reported that Lactobacillus acidophilus was a lactic acid-producing inhabitant of the human intestines. Numerous studies have claimed that Lactobacillus acidophilus preparations and milk fermented by Lactobacillus acidophilus have prophylactic and therapeutic properties $(3,6-9$, $12,13)$. The interest in this idea has increased along with a growing awareness of the importance of a healthy (balanced) microflora. Traditionally, a beneficial role has been assigned to $L$. acidophilus. However, Katelaris et al. (20) reported that $L$. acidophilus strain LA, like $L$. fermentum strain KLD, did not have any protective effect against diarrhea. This controversy still remains to be solved. In 1992, Fujisawa et al. (11) reported that the Lactobacillus strains identified as Lactobacillus acidophilus according to their biochemical characteristics, including carbohydrate fermentation pattern and isomer of lactic acid, could be divided into two major clusters which were in turn subdivided into six smaller

*Corresponding author. Mailing address: Microbiology Group, Technology and Research Institute, Snow Brand Milk Products Co., Ltd., 1-1-2, Minamidai, Kawagoe, Saitama 350-1165, Japan. Phone: +81-49-242-8161. Fax: +81-49-242-8696. E-mail: ht4s-fjwr@asahi-net.or.jp clusters (Lactobacillus acidophilus complex: LAC) which were given species status as $L$. acidophilus (A1), L. crispatus (A2), L. amylovorus (A3), L. gallinarum (A4), L. gasseri (B1), and L. johnsonii (B2). This may, at least partly, clarify the confusion over the anti-infectious ability of $L$. acidophilus.

There is very limited knowledge about the species of LAC responsible for the beneficial role in the human gastrointestinal tract. Colonization is an important property of beneficial bacteria entering the gastrointestinal tract. It is important that bacteria adhere, at least transiently, to the intestinal epithelium to colonize the intestine. If the bacteria do not reach and adhere to the intestine, they are not likely to influence the intestinal balance. Thus we focused our interest on the difference regarding colonization abilities, among six species of LAC. It is likely that the intrinsic capacity for survival and colonization differs among the species of LAC; however, there is very little information available at present in this respect. In order to achieve our objectives, we have to survey the population of human fecal Lactobacillus flora as the first step.

From the results of reexaminations of Lactobacillus acidophilus stock cultures of human fecal origin which had been identified phenotypically, $L$. gasseri was the predominant species $(11,18,19,22)$. However, in general, there are two major difficulties in the isolation of fecal lactobacilli. One is the suppression of lactobacilli 
growth on selective media. The other refers to the selection, or isolation, of lactobacilli grown on non-selective media used for fecal flora analysis (24). LBS agar is a well-known selective medium for isolating lactobacilli; however, lactobacilli growth on the plates is often suppressed. In addition, Gilliland et al. (12) reported that most colonies formed on original LBS agar under anaerobic conditions (GasPak system, BBL, Cockeysville, $\mathrm{MD}:\left(10 \% \mathrm{H}_{2}, 10 \% \mathrm{CO}_{2}\right.$, and $\left.\left.80 \% \mathrm{~N}_{2}\right)\right)$ had characteristics of Bifidobacterium species. It is questionable whether the library of Lactobacillus strains which has been constructed from isolates from the medium reflects the proportion of original fecal Lactobacillus flora. Moreover, BL agar is often used to isolate fecal bacteria including lactobacilli. However, isolation of lactobacilli from the medium is quite difficult because of the growth of a huge number of strains of other major anaerobic species. Therefore, the library of Lactobacillus strains constructed by isolates from these media seems to be inappropriate for the examination of fecal Lactobacillus flora. In this article, we developed a two-step lactobacilli isolation method to construct a stock library.

The aim of the present study was to determine which are the dominant species of Lactobacillus acidophilus complex in human fecal samples.

\section{MATERIALS AND METHODS}

Examination of the culture system for isolating lactobacilli from fecal specimens. Four commercially available media, namely BL agar (Nissui Pharmaceuticals Co., Ltd., Tokyo, Japan), Lactobacilli MRS agar (DIFCO, Detroit, MI), LBS agar (Becton Dickinson, Cockeysville) and LBS broth (Becton Dickinson), were examined to establish a method for isolating lactobacilli from fecal samples. Three types of $\mathrm{CO}_{2}$ generators, AnaeroPack $\mathrm{CO}_{2}$ (Mitsubishi Gas Chemical Co., Inc., Tokyo: a limited aerobic $\left(15 \% \mathrm{O}_{2}, 5 \% \mathrm{CO}_{2}\right.$, and $80 \% \mathrm{~N}_{2}$ )), AnaeroPack Campylo (Mitsubishi Gas Chemical Co., Inc.: a limited anaerobic $\left(10 \% \mathrm{O}_{2}\right.$, $10 \% \mathrm{CO}_{2}$, and $80 \% \mathrm{~N}_{2}$ )), and AnaeroPack Anaero (Mitsubishi Gas Chemical Co., Inc.: strict anaerobic $\left(20 \% \mathrm{CO}_{2}\right.$ and $\left.80 \% \mathrm{~N}_{2}\right)$ ), were evaluated. To establish the optimal lactobacilli isolation process, isolation was performed using a combination of these media and gas generators. Furthermore, combinations of isolation steps were examined to optimize the isolation process.

Internal standard LAC strains used to evaluate recovery from fecal samples. Streptomycin-rifampicin resistant LAC strains were selected and used to estimate the recovery of LAC strains from fecal samples.
L. gasseri SBT2055SR, L. acidophilus SBT2062SR, L. crispatus SBT1264SR, L. amylovorus SBT1762SR, L. gallinarum SBT1739SR, and L. johnsonii SBT 2050SR were used as internal standard strains to evaluate the recovery.

Volunteers. Fifteen healthy volunteers participated in this study. All volunteers, between 23 and 48 years of age, were researchers or research assistants of Snow Brand Milk Products Co., Ltd. They did not have a history of gastrointestinal disorders or constipation. None of them had eaten fermented food such as fermented milk, pickled vegetables or "natto," a Japanese traditional food, nor had they taken any antibiotics during four weeks previous to the sampling of feces. All the volunteers agreed to participate in this study after being informed of the details of the study.

Established isolation protocol of lactobacilli. One gram of fresh fecal samples was homogenized using a Teflon homogenizer with an anaerobic diluter (19) and serially diluted 10 -fold. A $0.1 \mathrm{ml}$ aliquot of each diluted suspension was plated on MRS agar plates and incubated under limited anaerobic conditions at $37^{\circ} \mathrm{C}$ for 2 days to avoid the growth of anaerobic bacteria including Bifidobacterium. Bacterial colonies grown on the MRS agar plates were transferred onto optimized LBS agar with a piece of sterilized velvet or with sterilized tooth picks and incubated under limited anaerobic conditions for 2 days at $37^{\circ} \mathrm{C}$. In this way, facultative cocci contaminating lactobacilli colonies on MRS plates were eliminated. The resulting bacterial colonies were isolated and cultured in optimized LBS broth. Most of the bacterial strains grown in the optimized LBS broth were lactobacilli. Light microscopy and conventional staining were used to confirm that the isolates were rods, Gram positive and non-motile, and therefore most likely to be lactobacilli.

Identification of isolated lactobacilli strains. The resulting lactobacilli strains isolated from fresh human fecal samples were identified by a genetic method employing restriction patterns of the enzymatically amplified 16S rRNA gene $(14,25,26)$ as described by Joho and Hashiba (18). Briefly, the lactobacillus strains freshly isolated from fecal specimens of human volunteers were cultured in $1 \mathrm{ml}$ of MRS broth at $37^{\circ} \mathrm{C}$ for 1 day. Bacterial cells were harvested and washed twice in STM buffer (50 mM Tris-maleate containing $1 \mathrm{~m}$ sucrose, $\mathrm{pH} 6.5$ ) by centrifuging them at $7000 \times g$ for 10 min. Washed cells were stored at $-80^{\circ} \mathrm{C}$ until use.

The frozen cells were resuspended in $0.5 \mathrm{ml}$ of STM buffer, and treated with $\mathrm{N}$-acetylmuramidase-SG (Seikagaku Corporation, Tokyo) at a final concentra- 
tion of $10 \mu \mathrm{g} / \mathrm{ml}$ at $37^{\circ} \mathrm{C}$ for $30 \mathrm{~min}$. The bacterial cell lysates thus obtained were treated with SDS at a final concentration of $1 \%$. Proteins were eliminated by extraction with an equal volume of phenol/chloroform/ isoamyl alcohol $(25: 24: 1)$. The aqueous layer was washed with an equal volume of chloroform, then 20 $\mathrm{ml}$ of $0.5 \%$ Proteinase-K (Sigma Chemicals, St. Louis, MO) was added. Proteinase-K treatment was carried out at $37^{\circ} \mathrm{C}$ for $20 \mathrm{~min}$. The DNA fraction was recovered by cold ethanol precipitation; then it was dried and resuspended in sterilized water and stored at $-20^{\circ} \mathrm{C}$ until use.

Polymerase chain reaction (PCR) for amplification of the 16S rRNA gene was done according to the procedure described by Joho and Hashiba (18). The sequences of the forward and the reverse primers used were 5'-AGAGTTTGATCCTGGCTCAG-3' and 5'AAGGAGGTGATCCAGCCGCA-3', respectively. The 16S rRNA gene was selectively amplified by PCR in total volume of $100 \mu$ l, with 2.5 units of Taq DNA polymerase (Perkin Elmer, Cetus, Sweden), $50 \mathrm{~mm} \mathrm{KCl}$, $10 \mathrm{~mm}$ Tris- $\mathrm{HCl}$ ( $\mathrm{pH} 8.3$ ), $1.5 \mathrm{~mm} \mathrm{MgCl}_{2} 0.01 \%$ (w/v) gelatin, $100 \mathrm{pmol}$ of each oligonucleotide primer and $200 \mu \mathrm{m}$ dNTP. The mixture was subjected to 36 cycles of the following incubations: denaturation at $94^{\circ} \mathrm{C}$ for $1 \mathrm{~min}$, annealing at $62^{\circ} \mathrm{C}$ for $2 \mathrm{~min}$ and chain extension at $72^{\circ} \mathrm{C}$ for $3 \mathrm{~min}$.

Amplified DNA was extracted with phenol/chloroform/isoamyl alcohol $(25: 24: 1)$, precipitated with 6 volumes of cold ethanol and $1 / 5$ volume of $3 \mathrm{M} \mathrm{KCl}$, and then rinsed with $70 \%$ ethanol. Precipitated DNA was dried and dissolved in $10 \mathrm{ml}$ of sterilized water.

An aliquot of $0.1-0.5 \mu \mathrm{g}$ DNA in $10 \mu \mathrm{l}$ of the appropriate buffer was digested overnight at $37^{\circ} \mathrm{C}$ with 5 units of a restriction endonuclease. Digestion with Dra I and Hinf I was performed independently; the restriction fragments of each reaction were loaded on separate lanes, and then electrophoresed using a 4-20\% polyacrylamide gradient gel in Tris-borate buffer $(18 \mathrm{~mA}, 100$ min). Gels were stained with Silver Stain Kit (Bio-Rad, Hercules, CA) and photographed. $\lambda$ DNA fragments digested with Eco RI and Hind III and pBR329 fragments digested with Hae III were used as molecular size markers.

Six other species of Lactobacillus, namely $L$. paracasei, $L$. salivarius, $L$. reuteri, $L$. brevis and $L$. plantarum, are also considered to be identifiable with the PFLP method described above. However, isolates classified to these species by this method were reidentified with the DNA-DNA hybridization method. Isolates with other fragment patterns with the RFLP method were defined as unidentified strains here. The unidentified strains with the same alphabetical code showed the same DNA fragment patterns.

Statistics. Statistical analyses were done with StatView ver 4 (SAS institute Inc., Cary, NC), with $\chi$ square test for homogeneity of LAC distribution. The level of significance was set at $p<0.05$.

\section{RESULTS}

\section{Establishment of Lactobacilli-Isolation Protocol from} Fecal Specimens

The effects of culture media and oxygen concentration in the culture systems on the selection of LAC strains and strains of major species in fecal anaerobic microflora were tested. The use of MRS agar and AnaeroPack Campylo did not affect the growth of the strains of 6 LAC species tested, while LBS agar prepared according to the manufacturer's instructions diminished the viable number of lactobacilli in fecal samples. The number of colonies of the LAC strains tested (2 strains per each species) grown on original LBS agar, was up to 10 times lower than the count obtained with MRS/Campylo. The declines were not LAC strain- or species-specific (Fig. 1). The count of viable LAC strains obtained with MRS agar under limited anaerobic conditions (MRS/Campylo culture) was comparable to that obtained with the conventionally strict anaerobic culture method (that is, with BL agar and steel wool jars (Hirayama Manufacturing Co., Ltd., Tokyo): data not shown). In addition, the MRS/ Campylo culture did not allow the growth of other strains of major anaerobic species of fecal origin (Fig. 2).

To eliminate the small number of colonies of cocci concomitantly grown in MRS/Campylo culture, we employed optimized LBS agar which was prepared by adding a half volume of glacial acetic acid according to the manufacturer's instructions $(0.66 \mathrm{ml} / \mathrm{l})$. The colonies grown on MRS agar were transferred to the optimized LBS agar; then the transferred plates were incubated under limited anaerobic conditions for another 2 days at $37^{\circ} \mathrm{C}$. Among the colonies isolated from fecal samples on MRS plates, 70 out of 261 colonies transferred from MRS to the optimized LBS could grow under limited anaerobic conditions $\left(10 \% \mathrm{CO}_{2}, 10 \% \mathrm{O}_{2}\right.$ and $80 \% \mathrm{~N}_{2}$ ). Of them, 62 were lactobacilli (identified on the basis of bacterial cell morphology after Gram staining).

Table 1 shows the recovery of streptomycin- and rifampicin-resistant strains of Lactobacillus acidophilus complex which had been premixed in human fecal 


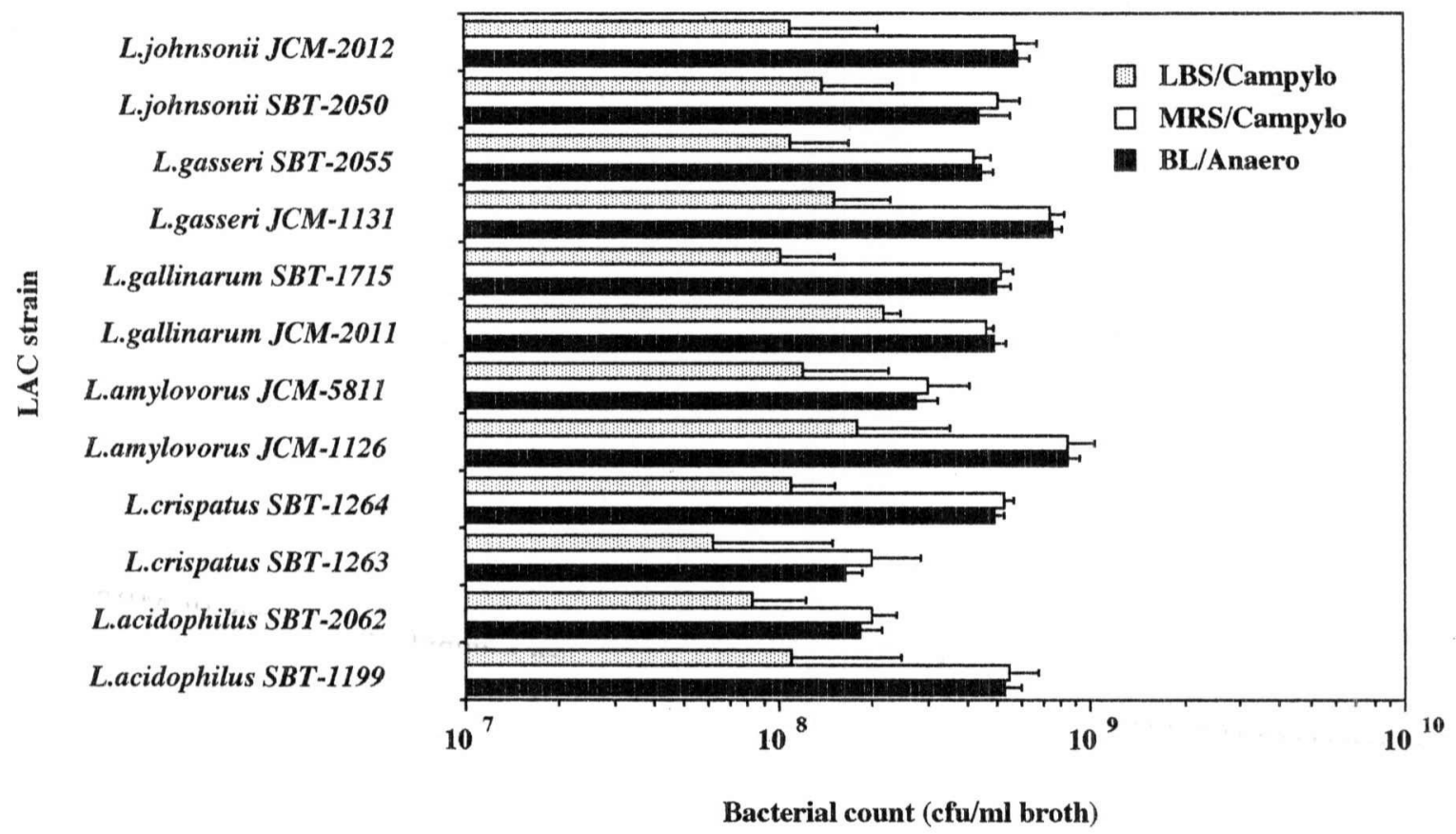

Fig. 1. Differences in recovery of strains of LAC with three different culture methods. MRS/Campylo: cultures were performed using MRS agar plates and AnaeroPack Campylo. LBS/Campylo: cultures were done using original LBS agar plates and AnaeroPack Campylo. BL/Anaero: BL agar plates and AnaeroPack Anaero were used for cultivation. Each column and bar represents the mean $\pm \operatorname{SD}(n=5)$.

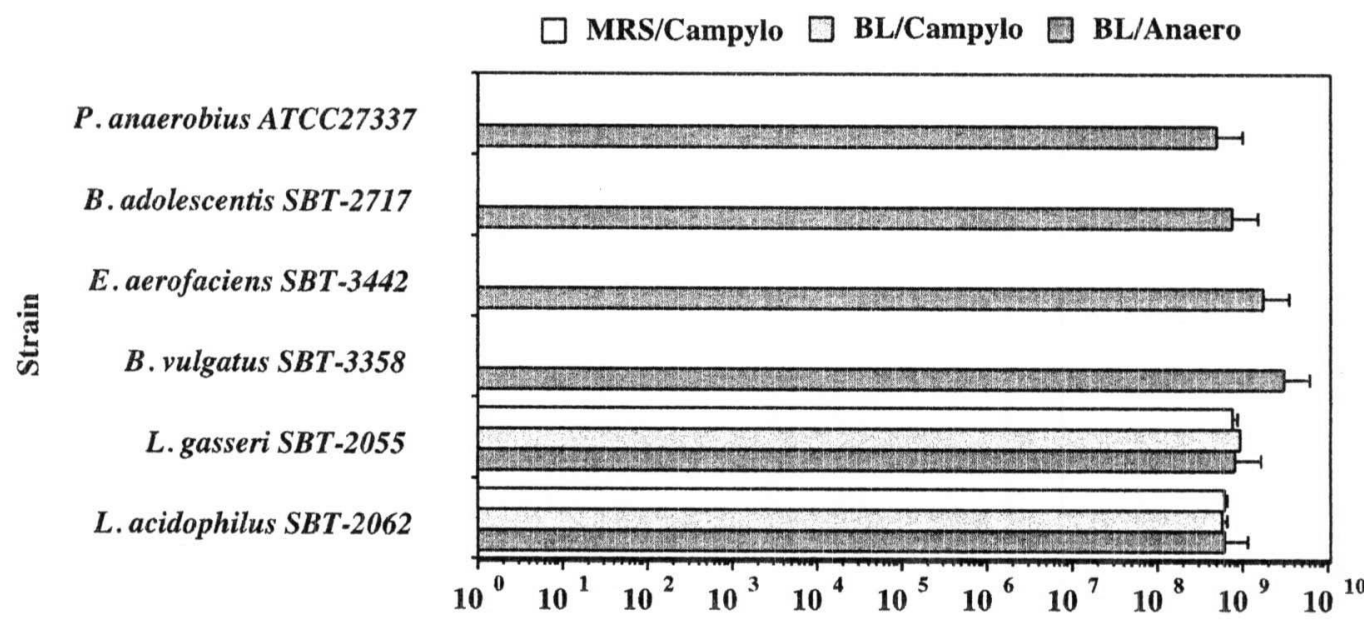

Bacterial count (cfu/ml broth)

Fig. 2. Recovery of strains of anaerobes often found in fecal specimens using three different culture methods. MRS/Campylo: the culture conditions were the same as described in the legend to Fig. 1. BL/Campylo: cultures were done using BL agar plates and AnaeroPack Campylo. BL/Anaero: the culture conditions were the same as described in the legend to Fig. 1. Each column and bar represents the mean $\pm \operatorname{SD}(n=5)$.

specimens. All of them were recovered from fecal specimens without significant losses. These results showed that the optimized LBS agar did not affect the number of colonies of lactobacilli formed. The combination of two culture systems proved an excellent strategy for the isolation of lactobacilli from feces.

The established protocol for the isolation of lactobacilli is summarized in Fig. 3. In brief, fresh fecal samples were weighed and homogenized using a Teflon homogenizer with an anaerobic diluter. The resulting suspen- 
Table 1. Recovery of streptomycin- and rifampicin-resistant variants of strains of Lactobacillus acidophilus complex using two different isolation methods from fecal specimens in which each variant strain had been premixed.

\begin{tabular}{lccc}
\hline & log number of bacteria & \multicolumn{2}{c}{ Recovery method } \\
\cline { 3 - 4 } Strains premixed in fecal sample & \begin{tabular}{c} 
added per g-feces \\
\cline { 3 - 3 }
\end{tabular} & MRS-SR/Campylo & $\begin{array}{c}\text { MRS-SR/Campylo }+ \\
\text { optimized LBS-SR/Campylo }^{\text {b) }}\end{array}$ \\
\hline L. acidophilus SBT2062SR & 7.05 & $7.108 \pm 0.285^{\mathrm{c})}$ & $7.029 \pm 0.206$ \\
L. crispatus SBT1264SR & 7.10 & $7.061 \pm 0.197$ & $7.031 \pm 0.235$ \\
L. amylovorus SBT1762SR & 7.15 & $7.203 \pm 0.417$ & $7.047 \pm 0.214$ \\
L. gallinarum SBT1739SR & 7.00 & $7.050 \pm 0.132$ & $6.904 \pm 0.287$ \\
L. gasseri SBT2055SR & 6.95 & $7.013 \pm 0.231$ & $6.857 \pm 0.227$ \\
L. johnsonii SBT2050SR & 6.96 & $6.981 \pm 0.175$ & $6.978 \pm 0.138$ \\
\hline
\end{tabular}

a) MRS-SR agar plates were employed as a selection agar and cultured under a limited anaerobic atmosphare using AnaeroPack Campylo, at $37^{\circ} \mathrm{C}$ for 2 days. The culture conditions were the same as those described in Materials and Methods, except that MRS-SR agar (MRS agar containing $1 \mathrm{mg} / \mathrm{g}$ of streptomycin and $10 \mu \mathrm{g} / \mathrm{g}$ of rifampicin) was used instead of MRS agar.

b) After MRS-SR/Campylo isolation, colonies grown on the medium were transferred to optimized LBS-SR plates and then cultured under the limited anaerobic atmosphare at $37^{\circ} \mathrm{C}$ for 2 days. Variant strains premixed in fecal sample were cultured using the MRS-SR/Campylo system and the colonies that grew on MRS-SR agar plates were transferred to optimized LBSSR agar plates (optimized LBS agar containing $1 \mathrm{mg} / \mathrm{g}$ of streptomycin and $10 \mu \mathrm{g} / \mathrm{g}$ of rifampicin). The transferred colonies were cultured again under limited anaerobic conditions at $37^{\circ} \mathrm{C}$ for 2 more days.

c) Data are expressed as the mean \pm SD of 5 separate examinations.

1) Isolated on MRS agar under a specific microaerobic atmosphare

2) Transfer to optimized LBS agar

3) Amplification of $16 \mathrm{~S}$ rRNA gene by PCR

4) PCR-RFLPs of amplified 16S rRNA gene

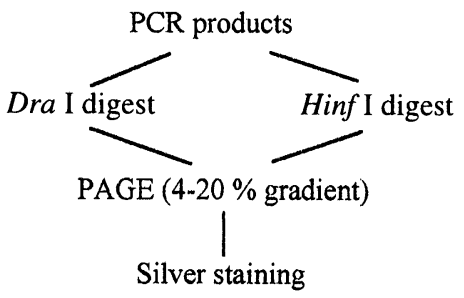

Fig. 3. Established protocol for the isolation and classification of fecal lactobacilli.

sion was serially diluted ten-fold with the anaerobic diluter. Aliquots of the dilutions were cultured. In the final step of isolation, 30 isolates, which were randomly chosen from the plates of one sample, were cultured in optimized LBS broth and the bacterial pellets were suspended in $30 \%$ glycerol in the broth and stored at -80 ${ }^{\circ} \mathrm{C}$ until identified.

\section{Distribution of Lactobacillus Acidophilus Complex in Human Feces}

Typical RFLP of LAC are shown in Table 2. Results of identification based on the RFLP of fecal LAC isolates were exactly the same as those obtained by DNA-
DNA hybridization method.

The distribution of LAC in fecal samples from volunteers is presented in Fig. 4. All the volunteers had at least one species of Lactobacillus in their feces. Five species of Lactobacillus other than four unidentified species were found in fecal Lactobacillus microflora; the most dominant species of Lactobacillus found in volunteers of this study was $L$. paracase $i$. This species was isolated from 12 out of 15 fecal samples. The average logarithmic number of $L$. paracasei in fecal specimens of volunteers was $6.780 \pm 0.702$ (per g; mean \pm $\mathrm{SD}, n=12$ ). LAC followed $L$. paracase $i$ in terms of frequency of occurrence. While LAC strains were isolated from 6 out of 15 volunteers in this study, LAC was dominant among isolated lactobacilli of LAC positive volunteers except for one volunteer.

Isolates of $L$. gasseri and isolates of $L$. johnsonii were recovered from 5 and 3 fecal specimens, respectively. $L$. amylovorus was the only species of cluster A LAC found in human fecal specimens in this study. Three out of 15 specimens contained L. amylovorus.

Lactobacillus flora of volunteers of blood type $\mathrm{O}$ seemed to be quite simple, with only 3 species other than LAC species (Fig. 4c: $p=0.0440$ ) being found in the fecal specimens; the major component was $L$. paracasei. On the other hand, Lactobacillus flora in fecal samples of volunteers of other blood types was respectively complex (Fig. 4b). Besides, LAC was the dominant Lactobacillus species in fecal samples of the volunteers whose blood was type $\mathrm{A}, \mathrm{B}$ or $\mathrm{AB}$. 
Table 2. Restriction fragment length polymorphic DNA fragment profiles of Dra I- and Hinf I-digested 16S rRNA-DNA of Lactobacillus acidophilus complex.

\begin{tabular}{|c|c|c|c|c|c|c|c|c|c|c|c|c|}
\hline \multicolumn{13}{|c|}{ L. acidophilus complex } \\
\hline & \multicolumn{2}{|c|}{$\begin{array}{l}\text { L. acidophilus } \\
\text { (A1) }\end{array}$} & \multicolumn{2}{|c|}{$\begin{array}{l}\text { L. crispatus } \\
\text { (A2) }\end{array}$} & \multicolumn{2}{|c|}{$\begin{array}{l}\text { L. amylovorus } \\
\text { (A3) }\end{array}$} & \multicolumn{2}{|c|}{$\begin{array}{l}\text { L. gallinarum } \\
\text { (A4) }\end{array}$} & \multicolumn{2}{|c|}{$\begin{array}{l}\text { L. gasseri } \\
\text { (B1) }\end{array}$} & \multicolumn{2}{|c|}{$\begin{array}{l}\text { L. johnsonii } \\
\text { (B2) }\end{array}$} \\
\hline \multicolumn{13}{|c|}{ Restriction fragment length (bp) } \\
\hline Restriction enzyme & Dra I & Hinf I & Dra I & Hinf I & Dra I & $\operatorname{Hinf} \mathrm{I}$ & Dra I & Hinf $\mathrm{I}$ & Dra I & $\operatorname{Hinf} \mathrm{I}$ & Dra I & $\operatorname{Hinf} \mathrm{I}$ \\
\hline \multirow[t]{17}{*}{ Type patterns } & a & I & a & II & b & II & a & III & $\mathrm{b}$ & IV & a & IV \\
\hline & & 948 & & 948 & & 948 & & 948 & & 948 & & 948 \\
\hline & 912 & & 912 & & & & 912 & & & & 912 & \\
\hline & & 840 & & 840 & & 840 & & 840 & & 840 & & 840 \\
\hline & 596 & & 596 & & $\begin{array}{l}731 \\
596\end{array}$ & & 596 & & $\begin{array}{l}731 \\
596\end{array}$ & & 596 & \\
\hline & & & & & & & & 412 & & 412 & & 412 \\
\hline & & & & 398 & & 398 & & & & & & \\
\hline & & 374 & & & & & & & & & & \\
\hline & & & & 339 & & 339 & & 339 & & 339 & & 339 \\
\hline & & 306 & & & & & & & & & & \\
\hline & & & & & 214 & & & & 214 & & & \\
\hline & & & & & & & & 191 & & & & \\
\hline & & 124 & & 124 & & 124 & & & & & & \\
\hline & & & & & & & & & & 84 & & 84 \\
\hline & & 80 & & 80 & & 80 & & & & 80 & & 80 \\
\hline & & 60 & & 60 & & 60 & & 60 & & & & \\
\hline & & & & & & & & & & 39 & & 39 \\
\hline
\end{tabular}

Restriction fragment length polymorphisms (RFLPs) of Dra I- and Hinf I-digested 16S rRNA gene are examined respectively on gradient gels (4-20\% polyacrylamide).

a) Overall $(n=15)$

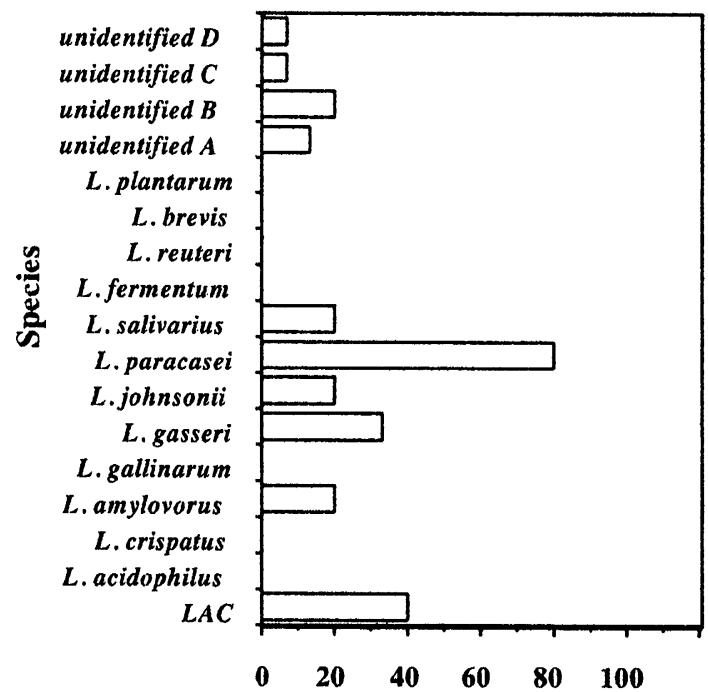

b) $\mathbf{A} / \mathbf{B} / \mathbf{A B}(n=10)$

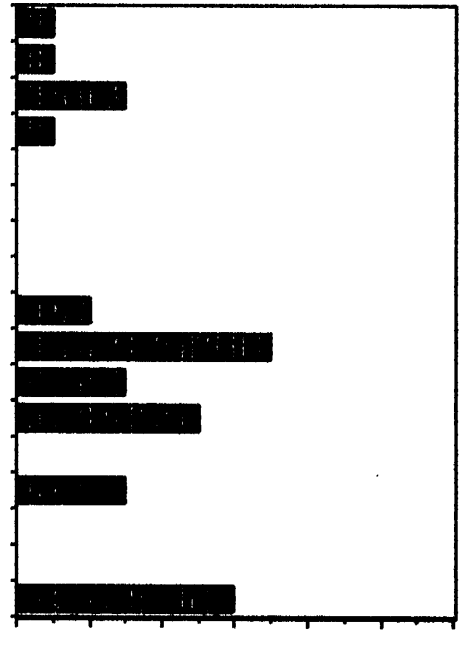

$\begin{array}{llllll}0 & 20 & 40 & 60 & 80 & 100\end{array}$

$\%$ occurrence c) $O(n=5)$

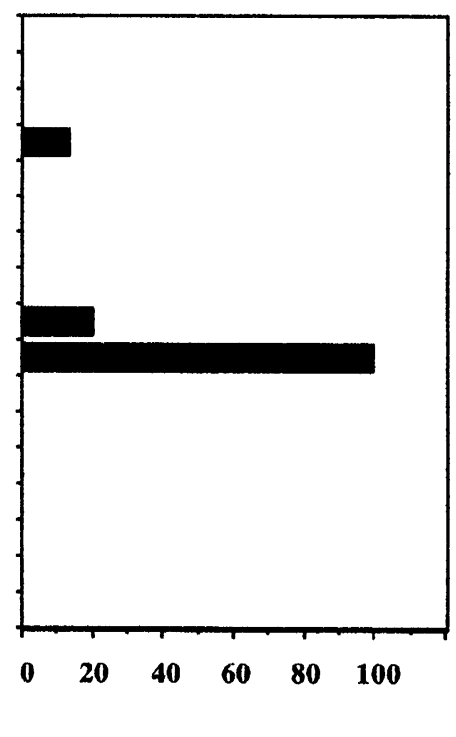

Fig. 4. Distribution of fecal Lactobacillus species in human fecal specimens. Overall: data from all fecal specimens. A/B/AB: data from fecal samples of volunteers who were of blood type A, $\mathrm{B}$ and $\mathrm{AB}$. O: data from fecal specimens of volunteers who were of blood type $\mathrm{O}$. 
a) Overall $(\mathbf{n}=15)$

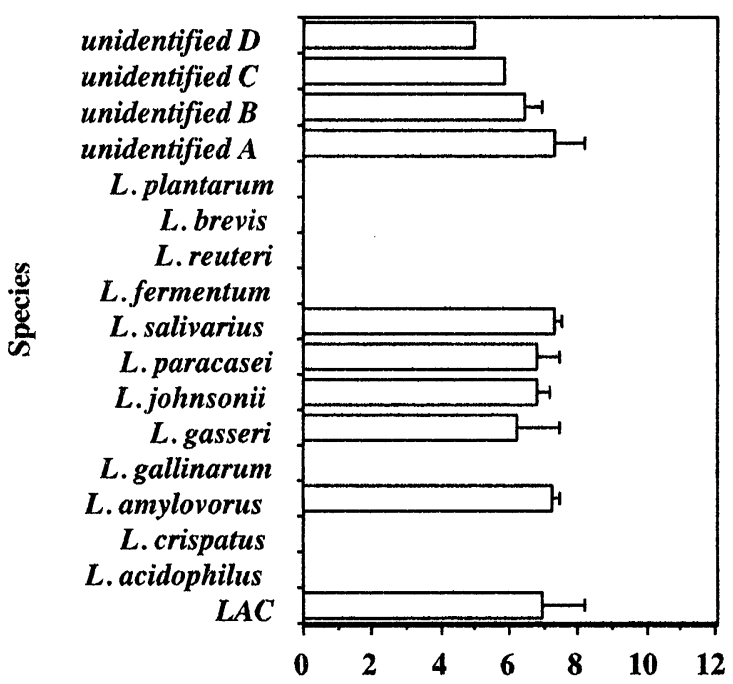

b) $\mathbf{A} / \mathbf{B} / \mathbf{A B}(\mathbf{n}=\mathbf{1 0})$

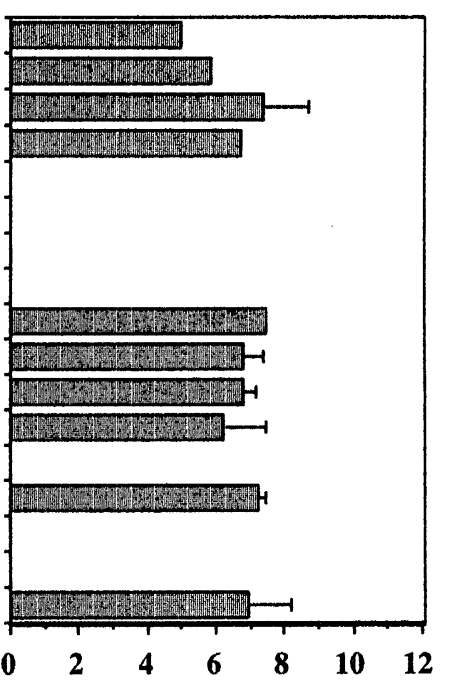

c) $\mathbf{O}(\mathbf{n}=5)$

\section{Bacterial count (log cfu/g-feces)}

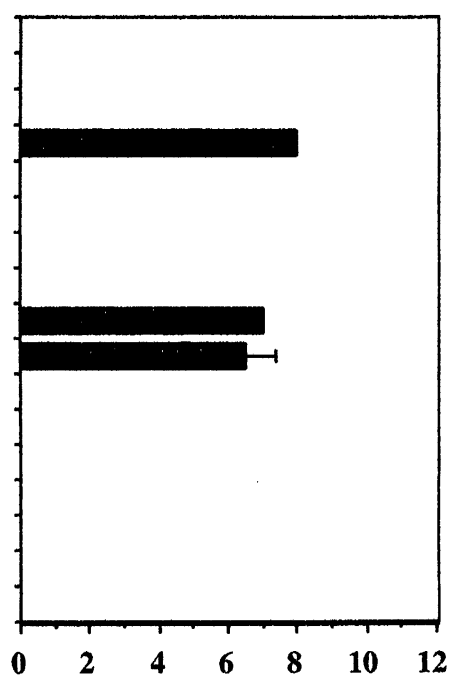

Fig. 5. Population of Lactobacillus species in human fecal specimens.

Overall: data from all fecal specimens. A/B/AB: data from fecal samples of volunteers who were of blood type A,

$\mathrm{B}$ and $\mathrm{AB}$. O: data from fecal specimens of volunteers who were of blood type $\mathrm{O}$.

Statistical analyses showed the distribution of Lactobacillus species in fecal samples was not homogenous in this study (Fig. 4: $p<0.0001$ ). Cluster B LAC occurred much more frequently than cluster A LAC $(p=0.0226)$. In addition, $L$. gasseri seemed to be the dominant species of LAC in fecal specimens of volunteers.

From the point of view of population, the number of viable cells of each Lactobacillus species in feces is shown in Fig. 5. The log number of fecal Lactobacillus was $7.133 \pm 0.723$ (per g; mean $\pm \mathrm{SD}, n=15$ ), while that of LAC species in feces of volunteers who had LAC strains varied from 4.30 to 7.72 (per $\mathrm{g} ; n=6$ ).

\section{DISCUSSION}

This study focuses on the composition of the Lactobacillus in feces of humans. It has been reported that $L$. acidophilus species is part of the human intestinal Lactobacillus flora (21). However, the L. acidophilus species is a very heterogenous group $(10,17)$. The study of Johnson et al. (16) revealed the genetic heterogeneity of the strains designated as L. acidophilus, on the basis of biochemical characteristics. The purpose of this study was to develop a simple protocol to isolate strains of Lactobacillus from human fecal specimens and to examine the fecal Lactobacillus flora of 15 volunteers using this newly developed method.
In evaluating the composition of Lactobacillus flora in human feces, the selection method for strains of Lactobacillus is of great importance. However, there has been no suitable isolation or selection method for lactobacilli because of reduction of their original number due to selective pressure. It is well known that LBS agar is a very good selective medium for lactobacilli. However, it is also well known that the conditions under which LBS agar is incubated greatly influence the recovery of LAC (12). Furthermore, Rogosa and Sharp (27) indicated that high concentrations of acetate caused by evaporation may inhibit some lactobacilli. In addition, incubation under strictly anaerobic conditions enables anaerobic Bifidobacterium species to grow and completely prevents the detection of LAC (12).

In order to reflect the original composition of the Lactobacillus population in feces, we improved the isolation method of lactobacilli from human fecal samples. In the first step, strict anaerobes including bifidobacteria could be eliminated by culture in an atmosphere of $10 \% \mathrm{CO}_{2}, 10 \% \mathrm{O}_{2}$, and $80 \% \mathrm{~N}_{2}$. Not all organisms detected on MRS/Campylo culture in this step were classified as typical Lactobacillus. Therefore, in the following step, acid resistant and facultative anaerobic rods were selected by replication from MRS agar plates to optimized LBS agar plates and cultured under limited anaerobic conditions. This step was important to elimi- 
nate facultative cocci that may have grown on MRS agar plates in the first step. The number of LAC colonies from a single LAC strain culture subsequently cultured on optimized LBS plates was almost the same as that of LAC grown on non-selective BL plates under strict anaerobic conditions.

In order to identify the isolates, an RFLP method for LAC identification, developed by Joho and Hashiba (18), was used to identify 12 species of fecal Lactobacillus. To date, several methods have been used to identify Lactobacillus strains present in the gastrointestinal microbiota $(1,15)$. The identification method is quite simple and rapid enough for the analysis to be completed within 2 days. In addition, results obtained with the RFLP method corresponded with the groupings obtained by DNA-DNA hybridization.

Using the two-step lactobacilli isolation protocol and RFLP identification, an appropriate analysis of human fecal Lactobacillus flora could be performed. This is a convenient and reliable method to analyze human fecal LAC flora. It could be applied to other analyses of Lactobacillus flora in samples of human origin, for example, that in viginal smears.

Joho and Hashiba (18) reported that 44 out of 68 strains of LAC of human fecal origin were L. gasseri, 7 were $L$. acidophilus, 8 were $L$. crispatus, 3 were $L$. amylovorus, 1 was $L$. gallinarum, and 5 were $L$. johnsonii. Kuroshima and Kodaira (22) reported that 15 out of 17 LAC strains were classified into L. gasseri, and the remaining 2 were classified into $L$. acidophilus. Fujisawa et al. (11) also surveyed the LAC composition of stock cultures and reported that 6 of 9 strains were $L$. gasseri, 2 were $L$. crispatus, and 1 was $L$. amylovorus. However, there is no evidence that the strains found in LAC culture stocks are completely independent of the others. The frequency of occurrence of L. gasseri in human fecal Lactobacillus flora followed that of $L$. paracasei. Our results basically agreed with those reported by other researchers who re-examined the composition of stock libraries of $L$. acidophilus of human fecal origin $(11,18,19,22)$.

It is interesting to note that no LAC species were isolated from fecal specimens of volunteers with blood type $\mathrm{O}$ at all. The distribution of isolates from volunteers with other blood types was highly complex. In volunteers with blood type $\mathrm{O}$, however, the cell counts of Lactobacillus species were lower and more homogenously distributed in the fecal specimens, indicating that the influence of blood type variation may be significant. Do blood group active antigens relate to the capability of LAC to colonize the human intestinal mucosa?
In animals, small intestinal glycolipid patterns including blood type glycosphingolipids vary among individuals as well as among species (4). At this point, further examination will be needed to clarify the mechanism(s) which underlie the phenomenon observed.

It can be concluded that LAC strains of human origin are mainly classified into cluster B; in particular, the frequency of isolation of L.gasseri from fresh fecal specimens of volunteers was higher than that of other LAC species. Thus, it appears that care must be taken in selecting strains of LAC for use in dietary preparations intended as a source of lactobacilli for establishment in the intestinal tract.

Carlsson and Gotherfors (5) reported that, in the human vagina, species of LAC account for most of the Lactobacillus flora. They also indicated that transmission of LAC from mother to child occurred at birth. More recently, Lachlak et al. (23) clarified that the dominant species of vaginal Lactobacillus of humans was L. gasseri. We found $L$. gasseri was the most frequently isolated species of LAC from human feces in this study. These facts may suggest that the development of intestinal Lactobacillus flora and the delivery of babies are closely related. Now we are interested in the composition of those of babies born by caesarean section.

\section{REFERENCES}

(1) Axelsson L, Lindgren S. 1987. Characterization and DNA homology of Lactobacillus strains isolated from pig intestine. J Appl Bacteriol 62: 433-440.

(2) Bibel DJ. 1988. Elie Metchnikoff's bacillus of long life. ASM News 54: 661-665.

(3) Blomberg L, Henriksson A, Conway PL. 1993. Inhibition of adhesion of Escherichia coli K88 to piglet ileal mucus by Lactobacillus spp. Appl Environ Microbiol 59: 34-39.

(4) Breimer ME, Hansson GC, Karlsson K-A, Leffler H. 1981. Blood group type glycosphingolipids from the small intestine of different animals analysed by mass spectrometry and thin-layer chromatography. A note on species diversity. J Biochem 90: 589-609.

(5) Carlsson F, Gotherfors L. 1975. Transmission of Lactobacillus jensenii and Lactobacillus acidophilus from mother to child at the time of delivery. J Clin Microbiol 28: 124 128.

(6) Chan RCY, Reid G, Irwin RT, Bruce AW, Costerton JW. 1985. Competitive exclusion of uropathogens from human uroepithelial cells by Lactobacillus whole cells and cell wall fragments. Infect Immun 47: 84-89.

(7) Chauviere G, Coconnier MH, Kerneis S, DarfeuilleMichaud A, Joly B, Servin AL. 1992. Competitive exclusion of diarrheagenic Escherichia coli (ETEC) from human enterocyte-like Caco- 2 cells by heat-killed Lactobacillus. FEMS Microbiol Lett 91: 213-218.

(8) Clements ML, Levine MM, Black RE, Robins-Browne RM, 
Cisneros LA, Drusano GL, Lanata CF, Saah AJ. 1981. Lactobacillus prophylaxis for diarrhea due to enterotoxigenic Escherichia coli. Antimicrob Agents Chemother 20: 104108.

(9) Coconnier MH, Bernet MF, Kerneis S, Chauviere G, Fournait J, Servin AL. 1993. Inhibition of adhesion of enteroinvasive pathogens to human intestinal Caco- 2 cells by Lactobacillus acidophilus strain LB decreases bacterial invasion. FEMS Microbiol Lett 110: 299-306.

(10) Fisher K, Johnson MC, Ray B. 1985. Lactose hydrolyzing enzyme in Lactobacillus acidophilus strains. Int J Food Microbiol 2: 23-29.

(11) Fujisawa T, Benno Y, Yaeshima T, Mitsuoka T. 1992. Taxonomic study of the Lactobacillus acidophilus group with recognition of Lactobacillus gallinarum sp. nov. and Lactobacillus johnsonii sp. nov. and synonymy of Lactobacillus acidophilus group A3 (Johnson et al., 1980) with the type strain of Lactobacillus amylovorus (Nakamura 1981). Int J Syst Bacteriol 42: 487-491.

(12) Gilliland ML, Speck ML, Nauyok GF Jr, Giesbrecht FG. 1978. Influence of consuming nonfermented milk containing Lactobacillus acidophilus on fecal flora of healthy males. J Dairy Sci 61: 1-10.

(13) Gilliland SE. 1989. Acidophilus milk products: a review of potential benefits to consumers. J Dairy Sci 72: 24832494.

(14) Irino K, Grimont F, Casin I, Grimont PAD. 1988. The Brazilian Purpuric Fever Study Group: rRNA gene restriction patterns of Haemophilus influenzae biogroup aegypticus strains associated with Brazilian purpuric fever. J Clin Microbiol 26: 1535-1538.

(15) Johansson JL, Quednau M, Ahrne S, Molin G. 1995. Lactobacillus plantarum by restriction endonuclease analysis of total chromosomal DNA using conventional agarose gel electrophoresis. Int J Syst Bacteriol 45: 670-675.

(16) Johnson JL, Phelps CF, Cummins CS, London J, Gasson F. 1980. Taxonomy of the Lactobacillus acidophilus group. Int J Syst Bacteriol 30: 53-68.

(17) Johnson MC, Ray B, Bhowmik T. 1987. Selection of Lactobacillus strains for use in "acidophilus products." Antonie
Leeuwenhoek 53: 215-223.

(18) Joho K, Hashiba H. 1992. Current status of taxonomy and development of rapid identification methods of lactic acid bacteria. Jpn J Dairy Food Sci 41: A207-A210 (in Japanese).

(19) Joho K, Nozaki N, Hashiba H, Toyoda S. 1994. Identification of Lactobacillus acidophilus complex species isolated from human feces by microplate hybridization methods. Snow Brand R\&D Reports 101: 209-221 (in Japanese).

(20) Katelaris PH, Salam I, Farthing MJG. 1995. Lactobacilli to prevent traveler's diarrhea. N Engl J Med 333: 13601361.

(21) Klaenhammer TR. 1982. Microbiological considerations in selection and preparation of Lactobacillus strains for use as dietary adjuncts. J Dairy Sci 65: 1339-1349.

(22) Kuroshima T, Kodaira S. 1983. Differentiation between Lactobacillus acidophilus and L. gasseri. Jpn J Bacteriol 38: 809-816.

(23) Lachlak N, Ageron E, Zampatti O, Michel G, Grimont PAD. 1996. Composition of the Lactobacillus acidophilus complex isolated from vaginal flora. Microbiologica 19: 123132.

(24) Mitsuoka T, Hayakawa K, Kitamura N. 1974. Die faekal Flora bei Menschen. II. Mitteilung: die Zuzammensetzung der Bifidobakterien-Flora der vershiedenen Altersgruppen. Zentralbl Bakteriol Parasitenkd Infektionskr Hyg I Abt Orig A 226: 469-478.

(25) Moreau P, Derclaye I, Gregoire D, Janssen M, Cornelis GR. 1989. Campylobacter species identification based on polymorphysm of DNA encoding rRNA. J Clin Microbiol 27: 2161-2166.

(26) Owen RJ, Beck A, Dayal PA, Dawson C. 1988. Detection of genomic variation in Providencia stuartii clinical isolates by analysis of DNA restriction fragment length polymorphisms containing rRNA cistrons. J Clin Microbiol 26: 2161-2166.

(27) Rogosa M, Sharp ME. 1959. An approach to the classification of the lactobacilli. J Appl Bacteriol 22: 329-340.

(28) Sandine WE. 1979. Roles of Lactobacillus in the intestinal tract. J Food Prot 42: 259-262. 